\title{
Efeito do Tratamento Hidrotérmico e Químico de Frutos de Banana 'Prata' no Controle da Antracnose em Pós-Colheita*
}

\author{
Claudia Sponholz ${ }^{1 * *}$, Ulisses G. Batista², Laércio Zambolim², Luiz C. C. Salomão³ \& Antônio A. Cardoso ${ }^{3}$ \\ 1Embrapa Meio-Norte, Av. Duque de Caxias 5650, CEP 64006-220, Teresina, PI, e-mail: claudia@cpamn.embrapa.br; \\ ${ }^{2}$ Departamento de Fitopatologia, Universidade Federal de Viçosa, CEP 36571-000, Viçosa, MG; ${ }^{3}$ Departamento de \\ Fitotecnia, Universidade Federal de Viçosa, CEP 36571-000, Viçosa, MG
}

(Aceito para publicação em 23/05/2004)

Autor para correspondência: Claudia Sponholz

SPONHOLZ, C., BATISTA, U.G., ZAMBOLIM, L., SALOMÃO, L.C.C. \& CARDOSO, A.A. Efeito do tratamento hidrotérmico e químico de frutos de banana 'Prata' no controle da antracnose em pós-colheita. Fitopatologia Brasileira 29:480-485. 2004.

\section{RESUMO}

Este trabalho objetivou testar a eficiência da hidroterapia e de alguns fungicidas no controle da antracnose causada por Colletotrichum musae e verificar o seu efeito na evolução da cor de frutos de banana (Musa spp.) 'Prata'. Buquês foram atomizados com C. musae $(2,5 \mathrm{x}$ $10^{6}$ esporos/ml em água) e imersos $24 \mathrm{~h}$ depois em água a $45^{\circ} \mathrm{C}, 50^{\circ} \mathrm{C}$ e $53^{\circ} \mathrm{C}$, durante $0,10,15$ e $20 \mathrm{~min}$. Outros buquês foram imersos por 3 min nos fungicidas tebuconazole, procloraz, difenoconazole e

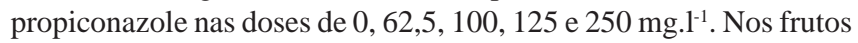
tratados a $45^{\circ} \mathrm{C}$ por $20 \mathrm{~min}$ (5, 10 ou 15 min foram ineficientes) a incidência da doença foi de um fruto infetado por buquê. A exposição dos frutos a $50{ }^{\circ} \mathrm{C}$ por 20 min reduziu a área lesionada em $85 \%$ e a 53 ${ }^{\circ} \mathrm{C}$ por 15 e $20 \mathrm{~min}$, os frutos apresentaram uma área lesionada de aproximadamente $3 \%$ e $0 \%$, respectivamente. Frutos não tratados apresentavam 53\% da área lesionada aos 12 dias de armazenamento. Os fungicidas procloraz em doses de 100, 125 e $250 \mathrm{mg} . \mathrm{l}^{-1}$, e propiconazole a $250 \mathrm{mg} . \mathrm{l}^{-1}$ foram os mais eficientes no controle da doença. Os frutos estavam sadios após 15 dias de armazenamento, enquanto que a testemunha apresentava aproximadamente $60 \%$ da área do fruto lesionada. Em armazenamentos de até 12 dias, o fungicida tebuconazole a $250 \mathrm{mg} . \mathrm{l}^{-1}$, procloraz a $62,5 \mathrm{mg} . \mathrm{l}^{-1}$ e propiconazole a 62,5, 100 e 125 mg. $\mathrm{l}^{-1}$ reduziram a área lesionada dos frutos para $1 \mathrm{a}$ $3 \%$. O fungicida difenoconazole foi ineficiente no controle da antracnose.

Palavras-chave adicionais: Musa spp., Colletotrichum musae, fungicidas, controle químico, hidroterapia, tratamento térmico.

\section{ABSTRACT}

Thermotherapy of banana 'Prata' to control post-harvest anthracnose

This study was done to determine the efficacy of hydrotherapy and some fungicides to control banana (Musa spp.) anthracnose caused by Colletotrichum musae and their effect on the color development in banana fruits of cultivar 'Prata'. Bunches of banana were sprayed with the conidial suspension $\left(2.5 \times 10^{6} / \mathrm{ml}\right)$ of the pathogen and 24 $\mathrm{h}$ later were immersed for $0,10,15$ or $20 \mathrm{~min}$ in hot water at $45^{\circ} \mathrm{C}, 50$ ${ }^{\circ} \mathrm{C}$ or $53^{\circ} \mathrm{C}$. Another group of inoculated bunches was immersed for three min in the suspension of tebuconazole, procloraz, difenoconazole or propiconazole at the concentrations of $0,62.5,100,125$ or 250 mg. $\mathrm{l}^{-1}$. After 12 days of storage, the disease incidence was only one fruit per bunch treated for $20 \mathrm{~min}$ at $45^{\circ} \mathrm{C}$, while treatment for less time periods were inefficient. The lesioned area was reduced to $15 \%$ when fruits were treated for $20 \mathrm{~min}$ at $50{ }^{\circ} \mathrm{C}$, but to $3 \%$ or $0 \%$ if treated for 15 or 20 minute at $53^{\circ} \mathrm{C}$, respectively compared to $53 \%$ in the non-treated fruits. The fungicides procloraz and propiconazole at a concentration of $100 \mathrm{mg} . \mathrm{l}^{-1}$ and $250 \mathrm{mg} . \mathrm{l}^{-1}$, respectively, were most efficient for disease control, since the fruits remained healthy even after 15-day storage, while the non treated fruits developed 60\% lesioned areas. On the other hand, the lesioned area varied from 1 to $3 \%$ after $12-$ day storage of fruits treated with tebuconazole (250 mg. ${ }^{-1}$ ), procloraz $\left(62,5{\left.\mathrm{mg} . \mathrm{l}^{-1}\right)}\right.$ or propiconazole $\left(62.5 \mathrm{mg}^{-\mathrm{l}^{-1}}\right.$ ). Difenoconazole did not control the disease.

\section{INTRODUÇÃO}

A antracnose, causada pelo fungo Colletotrichum musae (Berk \& Curtis) é a doença de pós-colheita mais importante em todas as regiões produtoras de bananas (Musa spp.) do mundo e responsável pela maioria das perdas. O fungo infeta os frutos ainda verdes e as infecções permanecem quiescentes até o amadurecimento, quando lesões escuras desenvolvem-se progressivamente (Abayasekara et al., 1998),

\footnotetext{
* Parte da tese de Mestrado do primeiro autor. Universidade Federal de Viçosa (2000)

***Bolsista do CNPq
}

afetando sua qualidade e comercialização.

Pulverizações ou banhos pós-colheita de fungicidas, especialmente benzimidazóis, são comumente usados para o controle da antracnose (Eckert \& Ogawa, 1985). Entretanto, o surgimento de isolados do fungo tem reduzido a efetividade destes produtos, substituindo-se pelo imazalil e pelo procloraz (Al Zaemey et al., 1993; Lapeyre De Bellaire \& Dubois, 1997).

A moderna agricultura requer redução no uso de pesticidas na produção de alimentos, havendo necessidade crescente de estratégias de controle alternativas (Lapeyre de Bellaire \& Mourichon, 1998).

Nos últimos anos, tratamentos físicos, químicos e 
Efeito do tratamento hidrotérmico e químico de frutos de banana ...

biológicos têm sido experimentados como alternativa aos fungicidas em pós-colheita, estendendo a vida comercial e preservando a qualidade de produtos hortícolas. Dentre esses tratamentos, destacam-se a luz UV, microrganismos antagonistas, sais de cálcio e o tratamento térmico.

O tratamento térmico é um método que tem a vantagem de ser livre de resíduos. Os patógenos podem ser controlados enquanto que o hospedeiro sofre pouca alteração. A eficácia do tratamento térmico sobre o patógeno é usualmente avaliada pela redução da viabilidade dos propágulos tratados (Golan \& Phillips, 1991), desordens fisiológicas durante o armazenamento (Jacobi \& Giles, 1997), consistência dos frutos, o que melhora a resistência a doenças (Couey, 1989).

Em bananas, a hidroterapia tem sido usada com propósitos de quarentena (Armstrong, 1982) e para estender a vida comercial do fruto (Domínguez, 1998).

A resistência de frutos de bananeira ao tratamento hidrotérmico foi determinada para o subgrupo Cavendish (AAA) (Rahman et al., 1994) e 'Prata de Santa Catarina' (AAB) (Reyes et al., 1998) para o controle da antracnose e da podridão da coroa respectivamente.

No Brasil há poucos trabalhos de controle químico e estudos de tratamento hidrotérmico para doenças de póscolheita de banana 'Prata'. Este fato tem levado produtores a utilizarem fungicidas empiricamente, sem registro de uso para estes fins. Este trabalho tem como objetivo avaliar a eficiência de alguns fungicidas sistêmicos, determinar um binômio de tempo e temperatura que controle a antracnose causada por $C$. musae e verificar os seus efeitos na evolução da cor de frutos de bananeira cultivar Prata.

\section{MATERIALEMÉTODOS}

Frutos de bananeira 'Prata', colhidos no estádio préclimatérico, em plantação comercial localizada no município de Viçosa, Minas Gerais, foram usados para o teste. As pencas de bananas foram selecionadas de cachos colhidos aleatoriamente, no índice de coloração 1 , descrito na escala de cores de Dadzie \& Orchard (1996), e com frutos de aproximadamente 34 mm de diâmetro.

Buquês contendo cinco frutos foram lavados com água, desinfestados com solução de hipoclorito de sódio a $0,2 \%$ de cloro ativo por $5 \mathrm{~min}$, enxaguados em água por duas vezes e pulverizados com uma suspensão de conídios de C. musae na concentração de 2,5x10 conídios $/ \mathrm{ml}$, obtida de colônias de dez dias de idade crescidas em Batata Dextrose Ágar + Estreptomicina (BDA+E). Para a pulverização, usou-se um atomizador De Villbs número 15. Outros buquês (Testemunha) foram inoculados apenas com água destilada esterilizada. Após a inoculação, os buquês foram incubados em câmara de nevoeiro por 24 h e submetidos à hidroterapia ou à quimioterapia.

\section{Tratamento hidrotérmico}

Para este procedimento, usou-se uma caixa d'água de amianto de $250 \mathrm{l}$, na qual foi instalado um sistema de aquecimento com quatro resistências elétricas de 1.500 watts, bomba d'água de $1 / 2 \mathrm{CV}$ e termostato. A temperatura da água foi medida com termômetro digital equipado com ponteira de aço inoxidável.

Os tratamentos consistiram na imersão de 16 buquês em $200 \mathrm{l}$ de água quente à $45^{\circ} \mathrm{C}, 50^{\circ} \mathrm{C}$ e $53^{\circ} \mathrm{C}$ durante $0,10,15$ e 20 min, seguido de resfriamento em água à temperatura ambiente por $5 \mathrm{~min}$. Para cada tratamento foram utilizadas quatro caixas contendo quatro buquês, cada uma servindo como uma repetição.

\section{Tratamento químico}

Doze buquês foram imersos por $3 \mathrm{~min}$ em $20 \mathrm{l}$ de soluções de cada um dos seguintes fungicidas: propiconazole 250 CE, difenoconazole 250 CE e tebuconazole 200 CE do grupo dos triazóis e no fungicida procloraz 450 CE do grupo imidazol, nas doses 0; 62,5; 100; 125 e 250 mg.l.-1. Um mililitro do adjuvante Triton X-100 foi adicionado à suspensão. Para cada tratamento foram utilizadas três caixas contendo quatro buquês, cada uma servindo como uma repetição.

\section{Avaliações}

Decorrido o tempo de imersão, os buquês foram retirados, deixados secar e acondicionados em caixas plásticas, forradas com uma camada de papel branco picado, e mantidos sob condições do ambiente $\left(11^{\circ} \mathrm{C}\right.$ a $\left.28{ }^{\circ} \mathrm{C}\right)$, até completo amadurecimento.

A cor dos frutos, a incidência e a severidade da doença foram avaliadas aos zero, três, seis, nove e 12 dias após os tratamentos (DAP). A incidência (I) foi determinada pelo número de frutos com sintomas da doença em cada buquê. A severidade (S) foi avaliada com auxílio de escala diagramática variando de 0 a $64 \%$ da área do fruto lesionada (Moraes, 1999). A evolução da cor dos buquês foi determinada com auxílio da escala visual de cores de Dadzie \& Orchard (1996), com índice de cor variando de 1 (totalmente verde) a 7 (totalmente amarelo com manchas castanhas).

\section{Delineamento experimental e análise estatística}

Os tratamentos foram dispostos num esquema de parcelas subdivididas no tempo, tendo nas parcelas o esquema fatorial $4 \times 5$ (quatro temperaturas e cinco tempos), para a hidroterapia; e 4x5 (quatro fungicidas e cinco doses), para a quimioterapia; e nas sub-parcelas os dias de avaliação (zero, três, seis, nove e 12), no delineamento inteiramente casualizado com quatro e três repetições respectivamente.

Foram desdobradas as interações tempos $\mathrm{x}$ temperaturas $\mathrm{x}$ dias de avaliação e fungicidas $\mathrm{x}$ doses $\mathrm{x}$ dias de avaliação, independente da sua significância, considerando como variável independente, no estudo da regressão, os dias de avaliação. Este fator foi decomposto em efeitos linear, quadrático, raiz quadrático, cúbico, cúbico base raiz quadrada, selecionando o modelo de efeito significativo, de significado biológico e com maior $\mathrm{R}^{2}$.

Os resultados obtidos foram previamente transformados em $\sqrt{x}$ ou $\sqrt{x+0,5}$, para efeito de análise estatística (Steel \& Torrie, 1980). As análises foram feitas pelo Sistema 
para Análises Estatísticas e Genéticas (SAEG).

\section{RESULTADOSE DISCUSSÃO}

Frutos tratados a $45^{\circ} \mathrm{C}$ não apresentaram diferenças entre os tempos de imersão no controle da doença, o percentual da área lesionada chegou ao máximo aos 12 dias após o tratamento (Figura 1a). O tratamento de $45{ }^{\circ} \mathrm{C}$ por $20 \mathrm{~min}$ apresentou, na média, apenas um fruto lesionado por buquê. Não houve efeito dos tratamentos com esta temperatura na evolução da cor dos frutos, e sua coloração aumentou normalmente ao longo do tempo, chegando ao índice sete aos 12 dias, contrastando com os resultados de Cabrera \& Domínguez (1998), obtidos com bananas 'Dwarf Cavendish'. O controle foi efetivo com temperaturas entre $45^{\circ} \mathrm{C}$ e $47,5^{\circ} \mathrm{C}$ durante 15 a 30 min e estes tratamentos estenderam a vida verde do fruto.

O percentual da área lesionada no tratamento a $50{ }^{\circ} \mathrm{C}$ foi menor no tempo de imersão de $20 \mathrm{~min}$, apresentando uma média de aproximadamente 15\% da área lesionada aos 12 dias após o tratamento. Aos 15 min de imersão, o percentual de área lesionada foi de 25\% e nos demais acima de 45\% (Figura 1b). Não houve diferença entre os tratamentos no amarelecimento dos frutos.

O tratamento mais efetivo $\left(53^{\circ} \mathrm{C}\right.$ e imersão acima de 10 min) reduziu a severidade da doença de $63 \%$ para menos de 3\% da área dos frutos lesionada (Figura 1c). Entretanto, frutos expostos a estes tratamentos, mostraram, nas extremidades, extensivo escurecimento da casca, a partir do sexto dia de avaliação. Frutos expostos à temperatura de $50{ }^{\circ} \mathrm{C}$ por $10 \mathrm{~min}$ ou mais apresentaram escurecimento a partir do nono dia de avaliação. Estes fatos evidenciam que a maturidade, a tolerância da cultivar ao calor e as condições ambientes antes da colheita devem ser consideradas quando do desenvolvimento ou aplicação de tratamentos térmicos pós-colheita (Golan \& Phillips, 1991).

Estudando os efeitos do tratamento térmico no amadurecimento, no metabolismo do etileno e na qualidade comercial para bananas 'Prata de Santa Catarina' e 'Dwarf Cavendish', Domínguez et al. (1998) reportaram que temperaturas abaixo de $50{ }^{\circ} \mathrm{C}$ causaram um atraso na evolução da cor da casca, sem afetar o acúmulo de sólidos solúveis e que temperaturas na faixa de $50{ }^{\circ} \mathrm{C}$ a $55^{\circ} \mathrm{C}$ causaram, em várias extensões, o escurecimento da casca, o acúmulo incompleto de açúcares solúveis e o aumento da sensibilidade ao "chilling” dos frutos. A produção de etileno foi progressivamente reduzida pelo aumento da temperatura, um efeito provavelmente mediado por uma diminuição da atividade da ACC oxidase na casca e na polpa. Temperaturas acima de $50{ }^{\circ} \mathrm{C}$ suprimiram o pico de evolução do etileno associado com o climatério e causaram uma dramática redução da atividade da ACC oxidase na casca. Resultados semelhantes foram obtidos por Cabrera \& Domínguez (1998) e Reyes et al. (1998).

As grandes limitações do uso do tratamento térmico são a falta de proteção residual contra a recontaminação por patógenos e as injúrias causadas nos frutos como o aumento
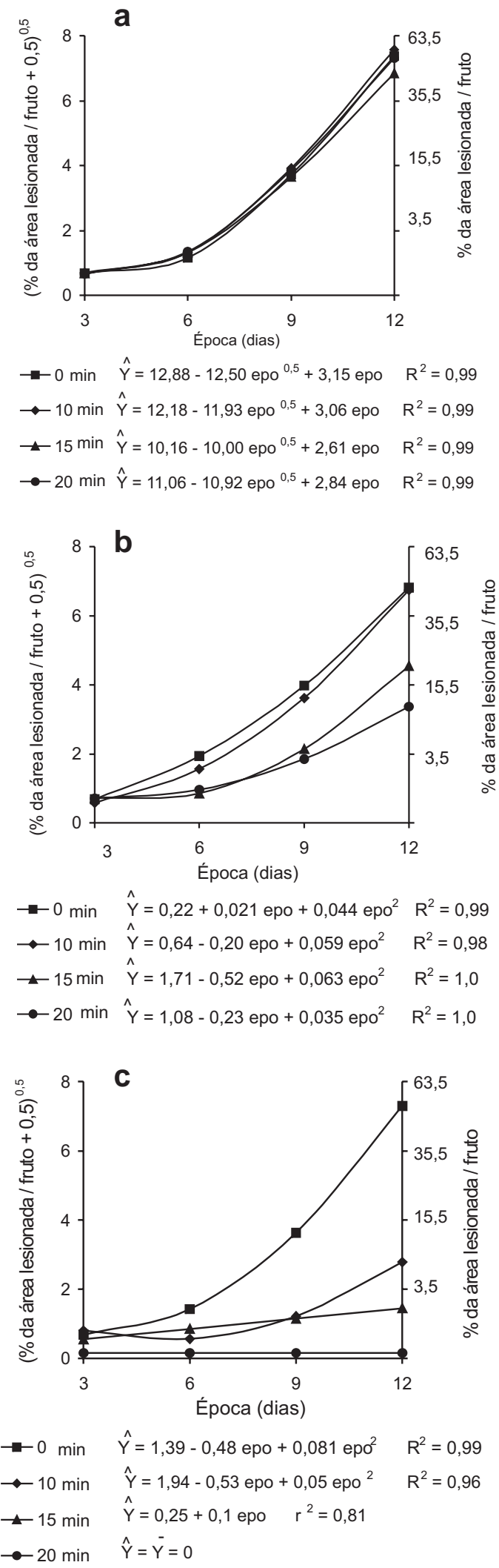

FIG. 1 - Porcentagem da área lesionada por fruto (dados transformados em $\sqrt{x+0,5}$ e destransformados) de bananeira (Musa spp.) 'Prata' inoculado com Colletotrichum musae e submetido a quatro tempos de imersão $(0,10,15$ e 20 min) na água com a temperatura a $45^{\circ} \mathrm{C}$ (a), a $50^{\circ} \mathrm{C}$ (b) e a $53^{\circ} \mathrm{C}$ (c), em função da época de avaliação. 
da perda de água, descoloração, aumento da suscetibilidade a microrganismos e redução da vida pós-colheita ou do período de armazenamento. Outros fatores como o amadurecimento, o metabolismo de açúcares, a produção de etileno, a produção de etanol, a atividade de enzimas pécticas e a perda de eletrólitos também podem ser afetadas (Golan \& Phillips, 1991).

Aos 12 dias após o tratamento os frutos imersos a $53^{\circ} \mathrm{C}$ por 20 min (Figura 1c) praticamente não apresentavam doença. Nos imersos por $15 \mathrm{~min}$, a severidade da doença foi baixa (aproximadamente 3\%), depreciando a comercialização.

Cabrera \& Domínguez (1998) relatam que o efeito do tratamento térmico é principalmente devido à diminuição da viabilidade dos esporos fúngicos, não descartando a possibilidade de um efeito combinado de indução de resistência nos frutos de banana.

A resposta do patógeno ao tratamento térmico pode ser influenciada pela umidade dos esporos, pela atividade metabólica do patógeno ou do inóculo, pela idade do inóculo, pela composição química e pela atividade da água no meio de tratamento (Golan \& Phillips, 1991). Segundo Baker (1962), mecanismos como desnaturação de proteínas, liberação de lipídios, destruição de hormônios, asfixia dos tecidos, redução das reservas de alimento, ou injúrias metabólicas com ou sem acúmulo de intermediários tóxicos podem estar envolvidos no controle de patógenos pelo calor.

Nos frutos tratados a $53{ }^{\circ} \mathrm{C}$ por tempos de imersão maiores que 10 min houve um pequeno efeito na evolução da cor até os nove dias de armazenamento e aos 12 dias todos estavam com a mesma cor, enquanto que frutos sem tratamento estavam em estádio mais avançado de senescência.

Os fungicidas procloraz em doses de 100, 125 e 250 mg. $\mathrm{l}^{-1}$, e propiconazole a $250 \mathrm{mg} . \mathrm{l}^{-1}$ foram os mais eficientes no controle da doença. Os frutos não apresentaram lesões após 15 dias de armazenamento, enquanto que frutos sem tratamento apresentavam aproximadamente 60\% da área do fruto lesionada (Figuras 2a e 2b). Estes fungicidas, nas doses avaliadas, foram

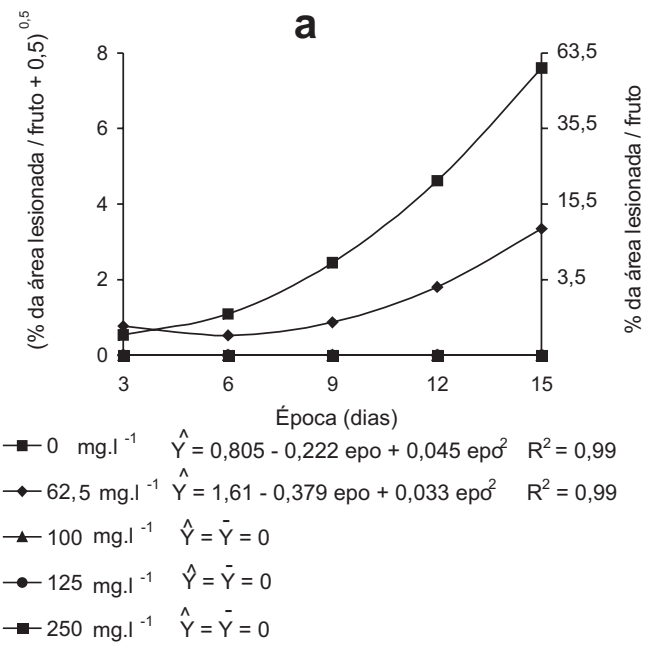

C

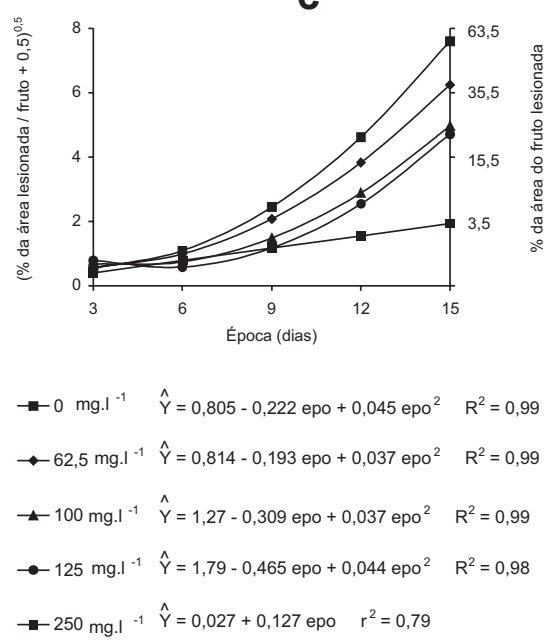

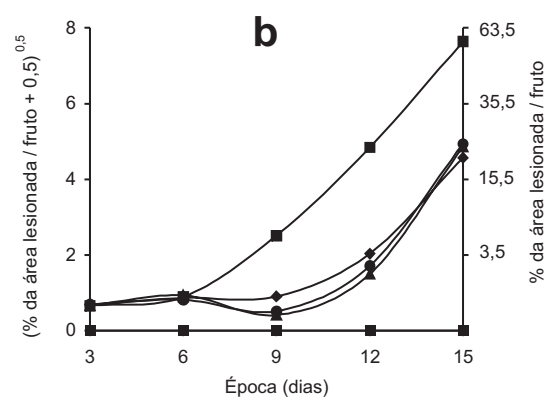

$\rightarrow-0$ mg.l $\mathrm{I}^{-1} \quad \hat{\mathrm{Y}}=8,835-8,291 \mathrm{epo}^{0,5}+2,061$ epo $\mathrm{R}^{2}=0,99$

$\rightarrow-62,5 \mathrm{mg} \cdot \mathrm{I}^{-1} \hat{Y}=-18,328+24,564 \mathrm{epo}^{0,5}-10,296 \mathrm{epo}+1,415 \mathrm{epo}^{1,5} \quad R^{2}=0,98$

$-100 \mathrm{mg}^{-1} \mathrm{I}^{-1} \hat{Y}=-35,834+46,189 \mathrm{epo}^{0,5}-18,779 \mathrm{epo}+2,47 \mathrm{epo}^{1,5} \quad R^{2}=0,96$

$\longrightarrow 125 \mathrm{mg} \cdot \mathrm{I}^{-1} \hat{\mathrm{Y}}=-28,758+37,834 \mathrm{epo}^{0,5}-15,671$ epo $+2,104$ epo ${ }^{1,5} \quad R^{2}=0,99$

$\rightarrow-250 \mathrm{mg} \cdot \mathrm{I}^{-1} \hat{\mathrm{Y}}=\overline{\mathrm{Y}}=0$

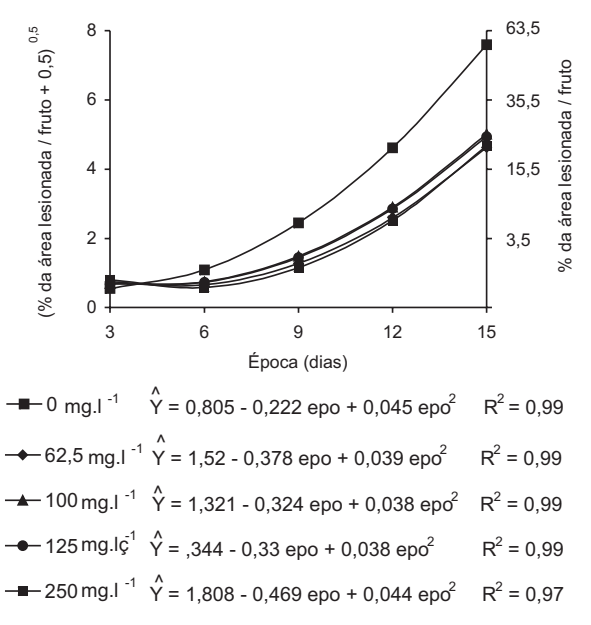

FIG. 2 - Porcentagem da área lesionada por fruto (dados transformados em $\sqrt{x+0,5}$ e destransformados) de bananeira (Musa spp.) 'Prata' inoculado com Colletotrichum musae e submetido ao tratamento com os fungicidas procloraz (a), propiconazole (b), tebuconazole (c) e difenoconazole (d) em função da dose e da época de avaliação. 


\section{Sponholz et al.}

capazes de controlar tanto as infecções induzidas artificialmente como as naturais vindas do campo, podendo ser recomendados como efetivos no controle da antracnose em pós-colheita em bananas 'Prata'. Em armazenamento em condições ambientes, mantiveram os frutos adequados para a comercialização até 15 dias após a colheita.

Estes resultados corroboram os de Moraes (1999) com os mesmos fungicidas. Resultados semelhantes foram também obtidos por Lapeyre De Bellaire \& Nolin (1994) com fungicidas inibidores da biosíntese do ergosterol, controlando C. musae em frutos inoculados artificialmente.

Os fungicidas, inibidores da biosíntese de esteróis, têm sido eficientes no controle de antracnoses em diversos frutos tropicais como: manga (Mangifera indica L.), abacate (Persea americana Mill) e mamão (Carica papaya L.) (Jeffries et al., 1990), e em lichias (Litchi chinensis Sonn) (Coates et al., 1994).

Alguns fungicidas sistêmicos podem erradicar a infecção que foi iniciada um ou mais dias antes do tratamento e esta habilidade vai depender do tempo decorrido entre a infecção e a aplicação do fungicida, da localização das estruturas de infecção quiescentes, e das propriedades de cobertura e penetração da formulação do fungicida (Johnson \& Sangchote, 1994).

Alguns frutos tratados com propiconazole, procloraz e difenoconazole apresentavam sintomas de toxidez na casca, sendo estes mais intensos nas doses mais elevadas, o mesmo

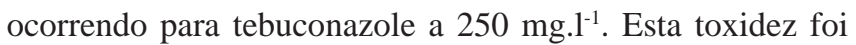
caracterizada por um escurecimento na casca principalmente em locais onde haviam injúrias mecânicas, causadas por falta de cuidados na colheita. Em experimento realizado por Moraes (1999), frutos tratados com procloraz a $125 \mathrm{mg} . \mathrm{l}^{-1}$ e com propiconazole em doses acima de 500 mg.l-1, também apresentaram sintomas de toxidez.

O fungicida tebuconazole a $250 \mathrm{mg} . \mathrm{l}^{-1}$ reduziu a área lesionada dos frutos de $60 \%$ para $3 \%$ aos 12 dias de armazenamento (Figura 2c), e a incidência foi de um fruto lesionado por buquê. Esse tratamento foi eficiente para frutos armazenados por tempos de até 12 dias. Resultado semelhante obteve-se com procloraz a 62,5 mg. $\mathrm{l}^{-1}$ e propiconazole a 62,5 ,

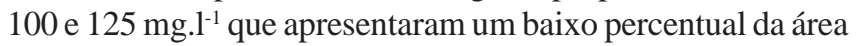
lesionada aos 12 dias (Figuras 2a e 2b), com uma incidência de menos de dois frutos contaminados por buquê para o procloraz e de um fruto lesionado por buquê para propiconazole a 100 e

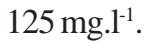

O fungicida difenoconazole não foi eficiente no controle da antracnose, mesmo nas maiores doses (Figura 2d), e permitiu uma alta incidência da doença (mais que três frutos por buquê infetados).

Nenhum dos tratamentos apresentou efeito na evolução da cor dos frutos. A evolução da cor foi semelhante para todos os fungicidas e doses usados, atingindo o índice 7 de coloração aos 12 dias após o tratamento. Um atraso da maturação e da coloração da casca de bananas tem sido relacionado com o uso de procloraz (Jones, 1991). No entanto, neste trabalho, este efeito não foi observado. Estes resultados diferem também dos obtidos por Moraes (1999), onde procloraz (125 mg. $\mathrm{l}^{-1}$ ) e propiconazole (250 mg.l-1 ${ }^{-1}$ mantiveram os frutos no índice de coloração 5, aos 12 dias após o tratamento, enquanto que frutos não tratados estavam no índice de cor 6.

\section{REFERÊNCIAS BIBLIOGRÁFICAS}

ABAYASEKARA, C., RATNAYAKE, S. \& ADIKARAM, N.K.B. Resistance of banana fruit to fungal disease: an overview. In: Jonson, G.I., Highley, E. \& Joyce, D.C. (Eds.) Disease resistance in fruit, Camberra: ACIAR Proceedings, n.80. 1998. pp.93-104.

AL ZAEMEY, A.B., MAGAN, N. \& THOMPSON, A.K. Studies on the effect of fruit-coating polymers and organic acids on growth of Colletotrichum musae in vitro and on postharvest control of anthracnose of bananas. Mycological Research 97:1463-1468. 1993.

ARMSTRONG, J.W. Development of a hot-water imersion quarentine treatment for hawaiian-grown ‘Brasilian' bananas. Journal of Economic Entomology 75:787-791. 1982.

BAKER, K.F. Thermoterapy of planting material. Phytopathology 52:1244-1255. 1962.

CABRERA, J.J.L. \& DOMÍNGUEZ, A.M. Use of hot water dips to control the incidence of banana crown rot. Acta Horticulturae 490:563569. 1998.

COATES, L.M., JOHNSON, G.I., SARDSUD, U. \& COOKE, A.W. Postharvest diseases of lychee in Australia, and their control. In: Jonson, G.I. \& Highley, E. (Eds.). Development of postharvest handling technology for tropical tree fruits, Australia: ACIAR Proceedings, n.58. 1994. pp.68-69.

COUEY, M.H. Heat treatment for control post-harvest disease and inset pest of fruits. HortScience 24:198-202. 1989.

DADZIE, B.K. \& ORCHARD, J.E. Post-harvest criteria and methods for routine screening of banana/plantain hybrids. IPGRI, INIBAP, 1996.

DOMÍNGUEZ, A.M., LÓPEZ CABRERA, J.J. \& GARCÍA, M.P. Effects of hot water tratments on postharvest quality and ethylene synthesis of bananas. Acta Horticulturae 490:529-535. 1998.

ECKERT, J.W. \& OGAWA, J.M. The chemical control of postharvest diseases: subtropical e tropical fruits. Annual Review of Phytopathology 23:421-454. 1985.

GOLAN, R.B. \& PHILLIPS, D.J. Postharvest heat treatment of fresh fruits and vegetables for decay control. Plant Disease 75:10851089. 1991.

JACOBI, K.K. \& GILES, J.E. Quality of 'Kensington' mango (Mangifera indica Linn.) fruit following combined vapour heat desinfestation and water disease control treatments. Postharvest Biology and Technology 12:285-292. 1997.

JEFFRIES. P., DODD, J.C., JEGER, M.J. \& PLUMBLEY, R.A. The biology and control of Colletotrichum species on tropical fruits crops. Plant Pathology 39:343-366. 1990.

JOHNSON, G.I. \& SANGCHOTE, S. Control of postharvest diseases of tropical fruits: challenges for the $21^{\text {st }}$ century. In: Champ, B.R., Highley, E. \& Jonson, G.I. (Eds.). Postharvest handling of tropical fruits, Camberra: ACIAR Proceedings, n.50. 1994. pp.140-161.

JONES, D.R. Chemical control of crown rot in Queensland bananas. Australian Journal of Experimental Agriculture and Animal Husbandry 31:693-698. 1991.

LAPEYRE DE BELLAIRE, L. de \& DUBOIS, C.. Distribution of Thiabendazole-resistant Colletotrichum musae isolates from 
Efeito do tratamento hidrotérmico e químico de frutos de banana ...

Guadeloupe banana plantains. Plant Disease 81:1378-1383. 1997.

LAPEYRE DE BELLAIRE, L. de \& MOURICHON, X. The biology of Colletotrichum musae (Berk. et Curt.) Arx and its relation to control of banana anthracnose. Acta Horticulturae 490:287-303. 1998.

LAPEYRE DE BELLAIRE, L. de \& NOLIN, J. Amérilation du contrôle du chancre sur les bananes d'exportation et traitements postrécolte. Fruits 49:179-185. 1994.

MORAES, W.S. Interação de métodos de controle de podridões em pós-colheita da banana 'Prata-anã' (AAB). (Tese de Doutorado). Universidade Federal de Viçosa, 1999.
RAHMAN, R.A., GRANDISON, A. \& CAMPBALL PLATT, G. Electron beam irradiation combined with hot-water immersion treatment for banana preservation. In: Champ, B.R., Highley, E. \& Jonson, G.I. (Eds.). Postharvest handling of tropical fruits, Australia: ACIAR Proceedings, v.50. 1994. p.378.

REYES. M.E.Q., NISHIJIMA, W. \& PAULL, R. Control of crown rot in 'Santa Catarina Prata' and 'Williams' bananas with hot water treatments. Postharvet biology and technology 14:71-75. 1998.

STEEL, R.G. \& TORRIE, J.H. Principles and procedures of Statistics. A Biometrical Approach. N.Y., Mc Graw-Hill Book Company. 1980. 